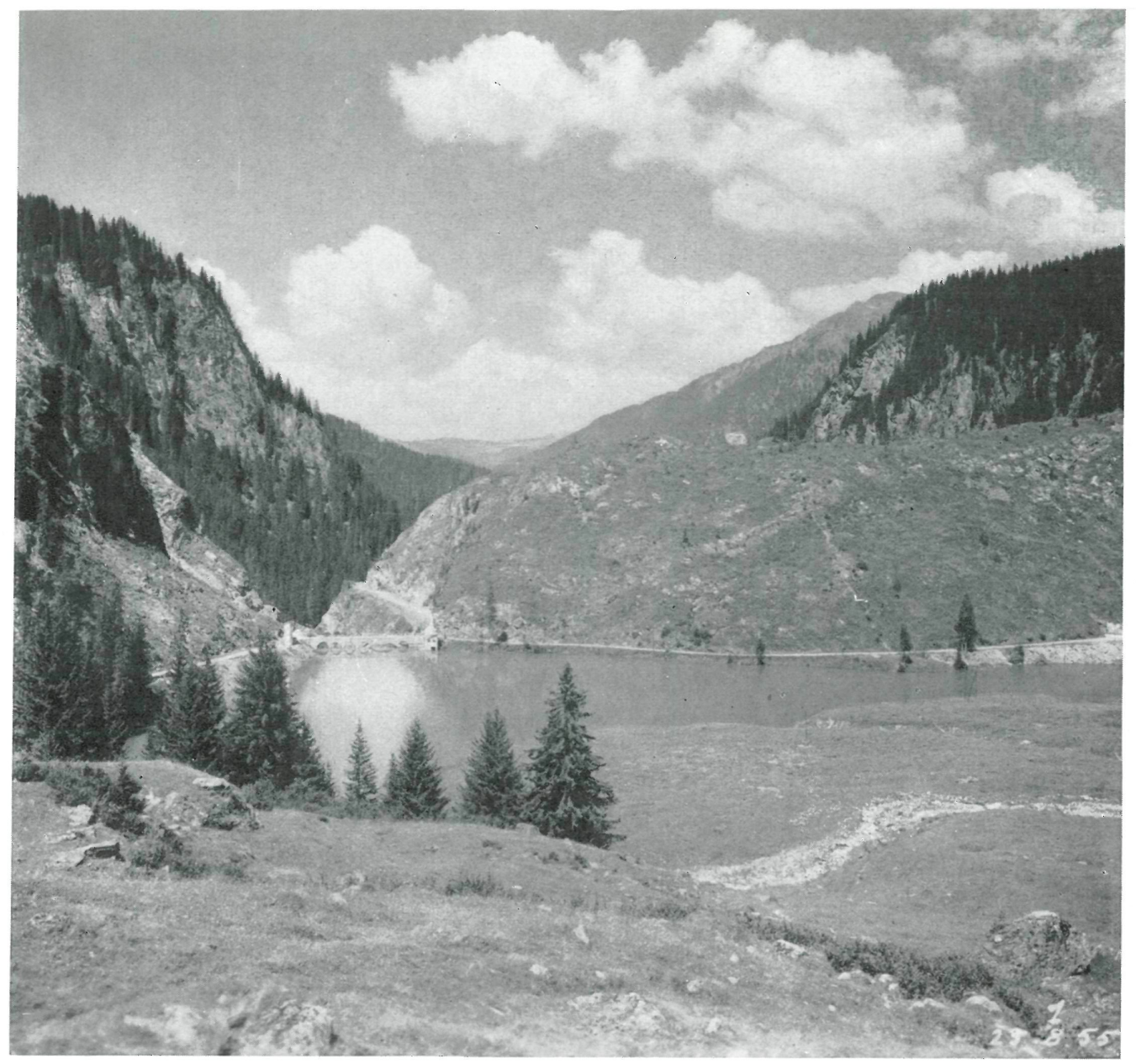

\title{
presa de Roselend
}

J. P. FREY y H. LEGROS, ingenieros del Gabinete Técnico Consultor Coyne y Bellier

simopesiss Esta presa se halla enclavada en el curso superior del rí, Doron de Beaufort, en los Alpes, en las proximidades del macizo del Mont Blanch. El embalse tiene uina capacidad de $18 \%$ hectómetros cúbicos.

Las aguas procedentes de la toma se llevan a la central subterránea a través de una galería de $13 \mathrm{~km}$ de longitud. La central tiene $500.000 \mathrm{~kW}$ de potencia instalada y aprovecha un salto de $1.208 \mathrm{~m}$ de caída y 50 obras, será del orden de $1.000 \times 10^{6} \mathrm{kWh}$, para cuya producción se contará con una cuenca de $22_{2}$ kilómetros cuadrados.

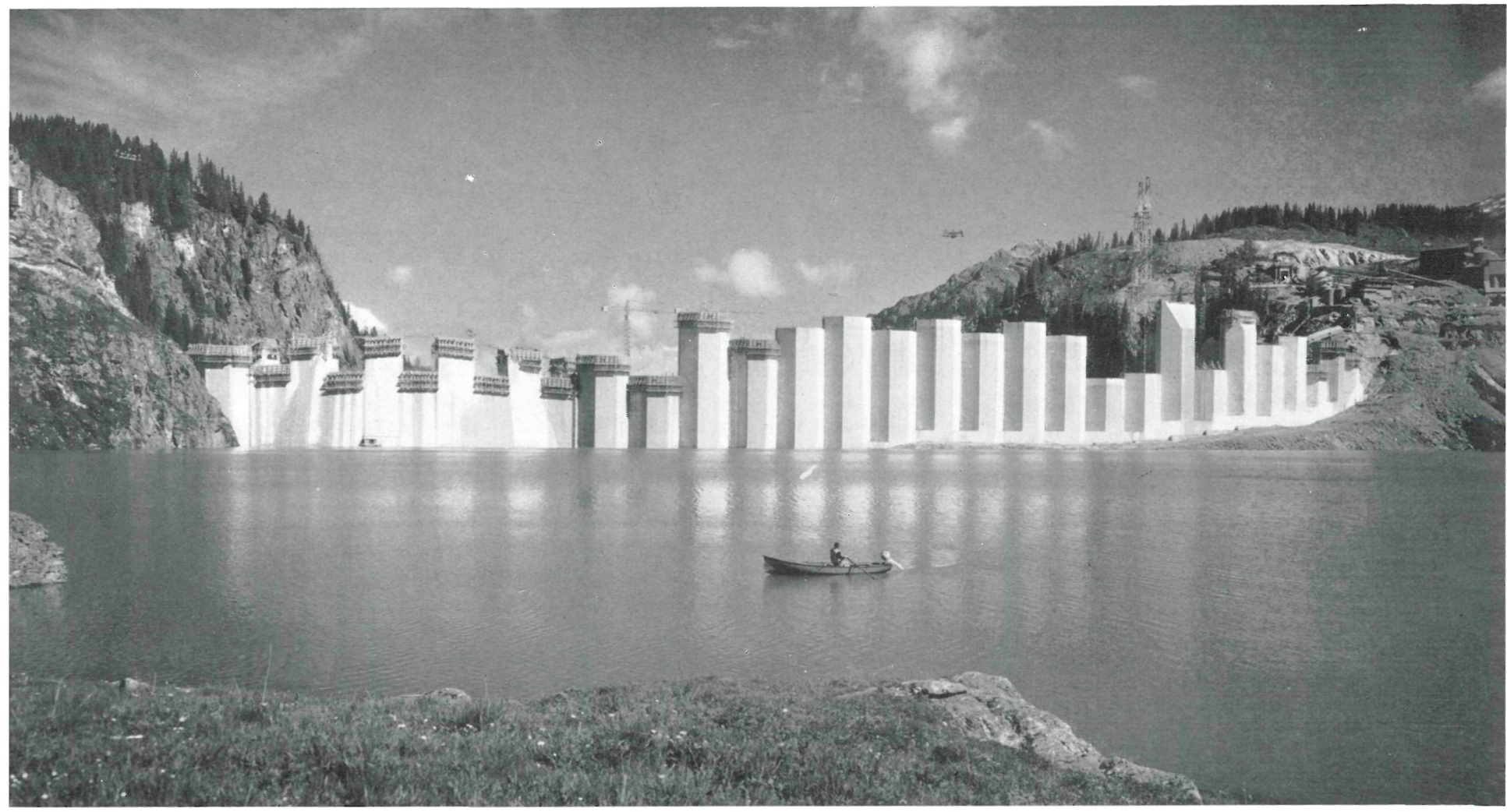




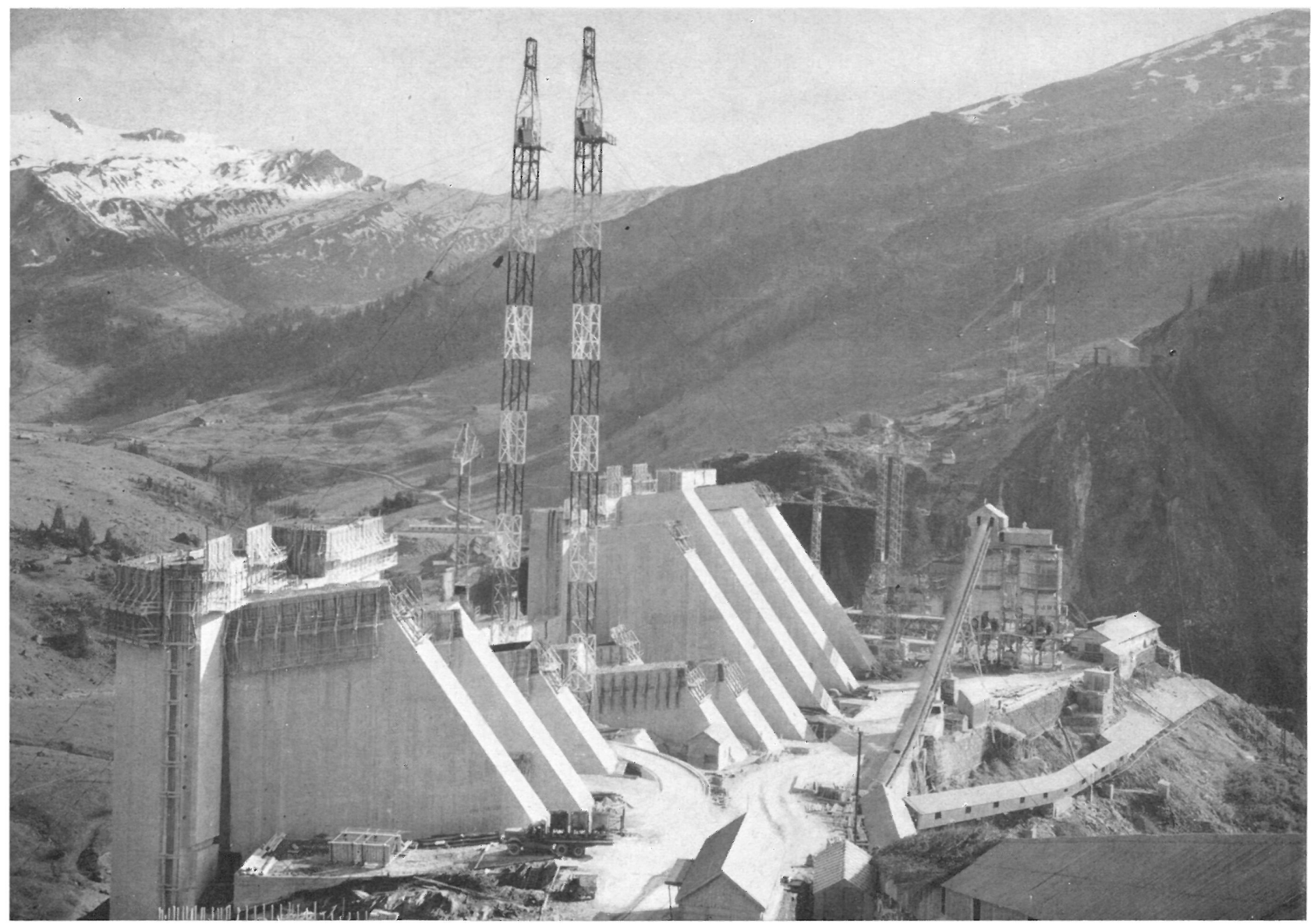

Dadas las condiciones locales, el cierre inicial suponía un dique de $150 \mathrm{~m}$ de altura. Estudiadas las soluciones presa de gravedad, aligerada o de contrafuertes y bóvedas múltiples, se eligió, como definitiva, la de un dique mixto de cierre que

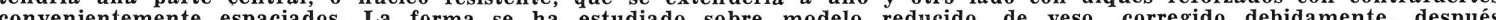
de cada ensayo, hastaciados. Lir el modelo ha deseado.

El paramento de aguas arriba es vertical. Los contrafuertes tienen un talud de 0,75 y un espesor máximo de 7,50 metros. En lo que respecta a la bóveda, el talud es de 1,05 en la dirección de la máxima pendiente. El espesor de la bóveda, en su base y en la parte correspondiente a la clave, es de 22 metros.

La presa tiene una longitud total de $820 \mathrm{~m}$, altura máxima de $150 \mathrm{~m}$ y el volumen de hormigón se eleva a 940.000 metros cúbicos.

\section{sitrucución gemeran}

El aprovechamiento hidroeléctrico denominado Roselend se halla situado en el tramo superior del río Doron de Beaufort, en los Alpes, en las proximidades del Mont Blanch. El dique de retención de aguas dará lugar a un embalse de 187 millones de metros cúbicos. El nivel máximo que alcanzarán las aguas embalsadas estará a 1.557 m sobre el nivel del mar.

Las aguas de este embalse se aprovecharán en una central subterránea, a la cual se conducirán a través de una galería de $13 \mathrm{~km}$ de longitud, en cuya extremidad se han instalado los grupos generadores con una potencia total de $500.000 \mathrm{~kW}$. El salto hidrostático aprovechable es de $1.208 \mathrm{~m}$ y el caudal disponible de $50 \mathrm{~m}^{3} / \mathrm{segundo}$

Una vez terminados los trabajos que constituyen el conjunto de este proyecto, de Electricité de France, la producción de energía eléctrica de esta central se elevará a 1.000 millones de $\mathrm{kWh}$. El conjunto del aprovechamiento se compone de distintos embalses y de numerosas aducciones que elevarán la superficie total de la cuenca superior a 272 kilómetros cuadrados.

\section{condiciomes locales}

Para el aprovechamiento óptimo de la capacidad de la cubeta de Roselend, era necesario cerrar el vaso con un dique de $150 \mathrm{~m}$ de altura, ya que si bien la margen izquierda de la garganta, que constituye el vaso, y hacia aguas abajo, presenta una pendiente pronunciada hasta el nivel deseado, la margen derecha, por el contrario, desaparece bruscamente a unos $90 \mathrm{~m}$ de altura relativa y se prolonga con pendiente suave muy lejos. 
Se pudo pensar en un cierre de gran longitud, dándole forma de presa de gravedad, aligerada o de bóvedas múltiples, pero la introducción de contrafuertes en la zona de esta garganta hubiese exigido dimensiones enormes que crearían dificultades de cimentación debido a las grandes pendientes.

Otra solución posible consistía en cerrar el vaso por medio de una bóveda tradicional, prolongada en la parte supe rior de la margen derecha por otro tipo de dique de cierre, pero el estribo necesario en esta margen, para hacer frente al empuje en la parte superior de la bóveda, hubiera alcanzado un volumen prohibitivo.

\section{In prescin}

La solución adoptada ha consistido en una bóveda de cierre de la garganta, concebida como un elemento local de cierre y resistente, continuado por un dique tipo gravedad, aligerado con algunos contrafuertes que se apoyan sobre la bóveda, la cual no era preciso que tuviera una coronación horizontal prevista para la cota de retención, por lo que enrasa oblicuamente y transmite los empujes directamente al terreno por medio de arcos que no necesitan apoyos particulares.

El dique de cierre, aligerado, está constituido por tramos de $20 \mathrm{~m}$ de luz. El paramento de aguas arriba es vertical, contrariamente a las técnicas ordinarias generales para este tipo de obras. La ubicación de la bóveda exigía, en efecto, que la coronación de la misma tuviese una posición muy avanzada, aguas arriba, respecto de su apoyo. Los contrafuertes forman un talud de 0,75 y tienen un espesor máximo de 7,5 metros.

La forma de la bóveda tiene una concepción completamente nueva, a la cual se ha llegado después de una seris de estudios sobre modelos reducidos de distintas formas, de las que 40 de ellas se han ensayado en 12 modelos de yeso, cada uno de los cuales se sometió a distintos retoques sucesivos. La bóveda construida tiene $22 \mathrm{~m}$ de espesor en $\mathrm{su}$ base y en la clave de ella. La coronación se ha enrasado según un plano con 1,5 de pendiente hacia aguas abajo. Los contrafuertes que se apoyan en la bóveda se han separado de ésta por medio de una junta. Estas juntas, impermeabilizadas, se han concebido de tal forma que se presten a todo movimiento diferencial relativo en las condiciones previstas.

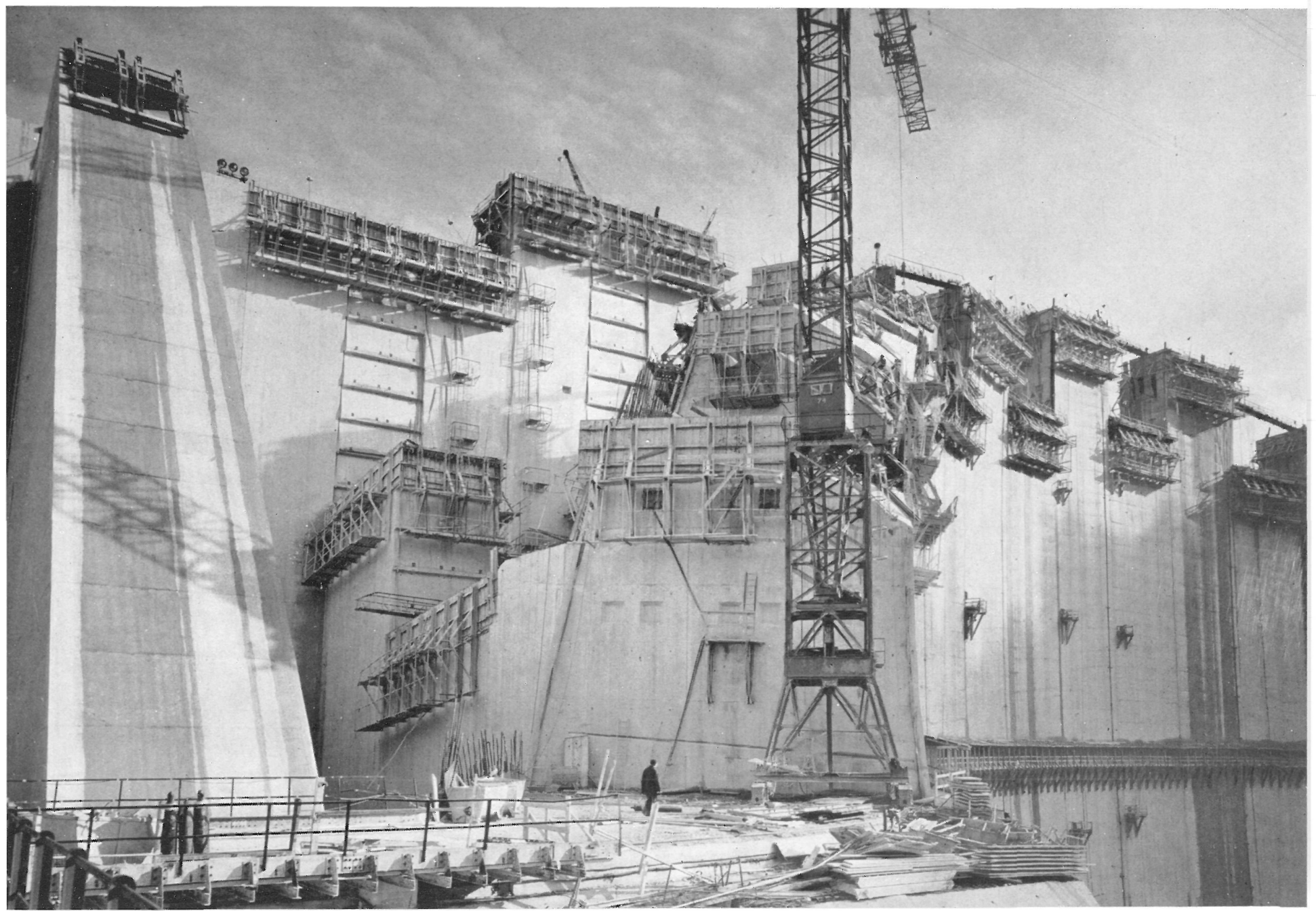



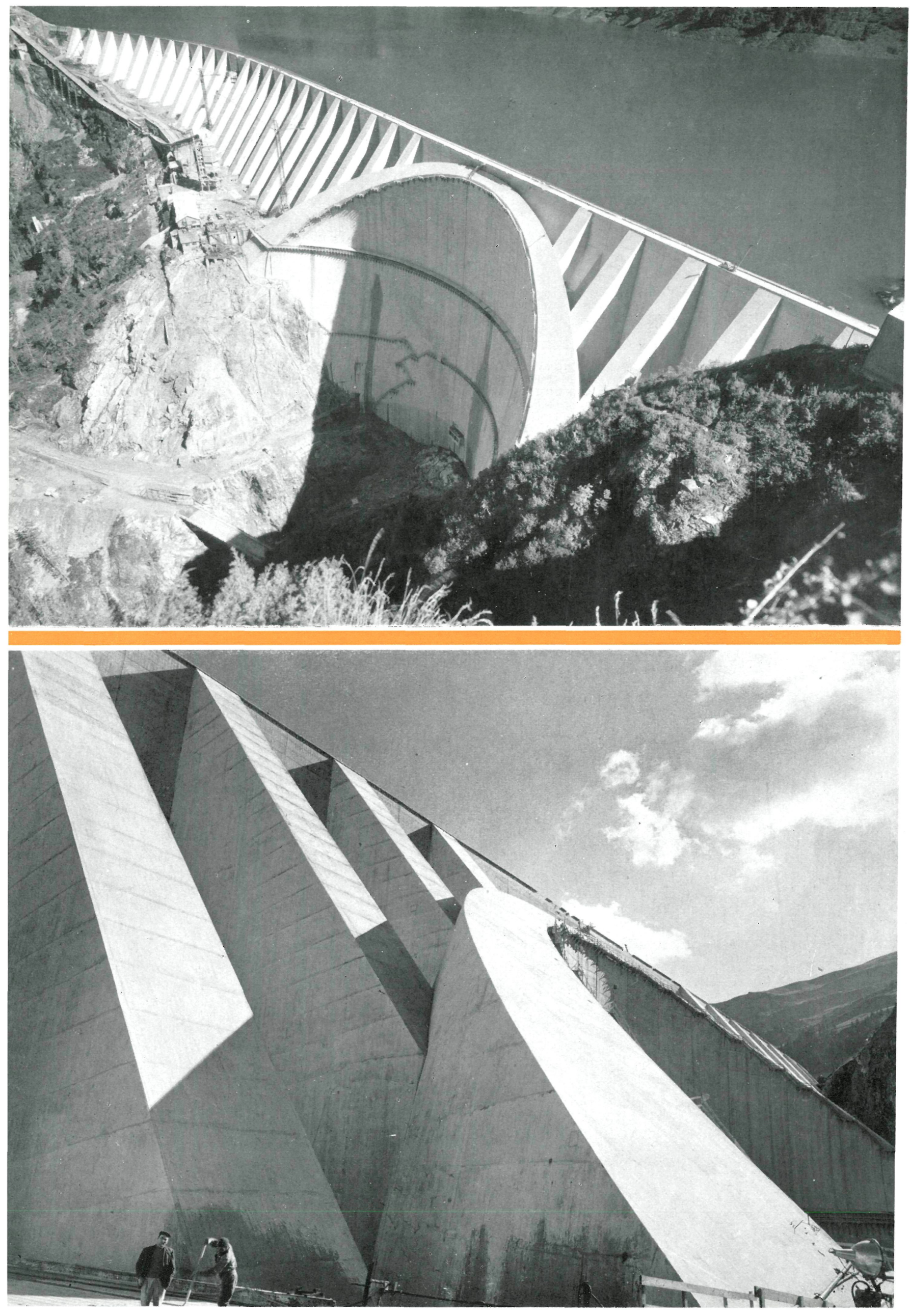


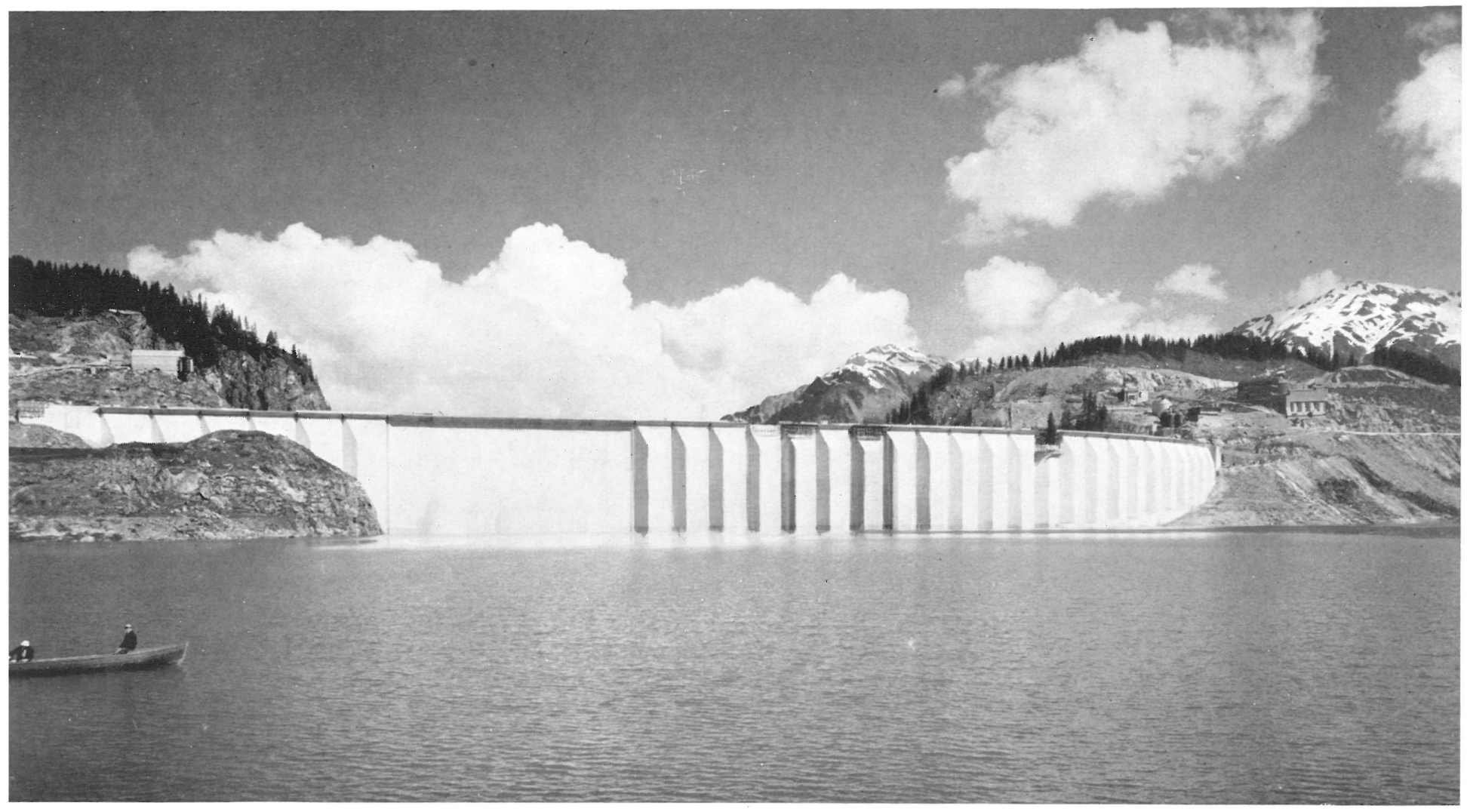

Fotos: H. BARANGER, Paris

La longitud total del dique mixto de cierre tiene un desarrollo, en la parte superior, de $280 \mathrm{~m}$, y la altura máxima, a partir de cimientos, es de $150 \mathrm{~m}$. El volumen total de hormigón empleado en su construcción se eleva a $940.000 \mathrm{~m}^{3}$.

\section{olburas ancerans}

La presa de Roselend no dispone de aliviadero de grandes avenidas, debido a la reducida extensión de la cuenca natural vertiente.

En la parte inferior de la presa, y en la zona correspondiente a la bóveda, se han previsto dos desagües distintos de fondo con una capacidad total de $90 \mathrm{~m}^{3} /$ segundo.

La toma de agua, completamente indepeidiente de la presa, se halla en la margen izquierda y no muy alejada aguas arriba de la misma.

\section{ejecureín}

Los trabajos preliminares de este aprovechamiento hidroeléctrico se empezaron en el año 1956 . La construcción, propiamente dicha de la presa, se ha efectuado durante seis períodos de seis meses, aproximadamente, que han tenido lugar durante los años de 1957 a 1962. El hormigonado a la altitud en que se halla la presa no ha hecho posible el aprovechamiento del año natural más que en su mitad.

Los áridos se obtuvieron de una cantera de esquistos cristalinos, que se explotó en la proximidad de las obras. Las centrales hormigoneras tenían una capacidad total de $270 \mathrm{~m}^{3} / \mathrm{h}$. El hormigón se colocó en obra por medio de grúas y dos blondines de 10 t y de $540 \mathrm{~m}$ de luz. El hormigón se colocó por tongadas de $50 \mathrm{~cm}$ de espesor, alcanzando alturas parciales de 1,5 de potencia.

La retención de aguas de esta presa empezó en el año 1960, es decir, bastante antes de haber terminado la presa y después de haber colocado en obra el 70 por 100 del total de hormigón.

El conjunto y disposición general de trabajos ha permitido pueda entrar en servicio la central generadora sólo cuatro años después del comienzo de los trabajos. 


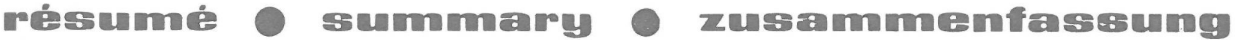

\section{Le burngerge Roselend}

J. P. Frey et H. Legros, ingénieurs.

Ce barrage est situé sur le cours supérieur du Doron de Beaufort, dans les Alpes, aux environs du massif du Mont Blanc. La retenue a une capacité de $18 \% \mathrm{~km}^{3}$.

Les eaux provenant de la prise sont amenées à la centrale souterraine par une galerie de $13 \mathrm{~km}$ de longueur. La centrale a $500.000 \mathrm{~kW}$ de puissance installée et utilise une chute de $1.208 \mathrm{~m}$ de hauteur et $50 \mathrm{~m}^{3} / \mathrm{m}$ de débit utilisable. Quand les travaux seront terminés, on disposera d'un bassin de $2^{r} 72 \mathrm{~km}^{2}$, qui permettra une production d'énergie électrique de l'ordre
de $1.000 .10^{3} \mathrm{~kW} / \mathrm{h}$.

Etant donné les conditions locales, la fermeture initiale supposait une digue de $150 \mathrm{~m}$ de hauteur. Après avoir étudié les solutions de barrage de gravité, barrage évidé ou à contreforts et à voûtes multiples, on a choisi, comme solution définitive, côté à l'aide de digues renforcées par des contreforts cortie centrale ou noyau résistant qui s'étendrait à l'un et à l'autre plâtre, dûment corrigé après chaque essai jusqu'à obtenir le modèle qui se comporta le mieux.

Le parement amont est vertical. Les contreforts ont une pente de 0,75 et une épaisseur maximum de 7,50.

En ce qui concerne la voûte, le pente est de 1,05 en direction de la pente maximum. L'épaisseur de la voûte, sur sa base et sur la partie correspondant à la clé, est de 22 mètres.

La longueur totale du barrage est de $820 \mathrm{~m}$, sa hauteur maximum de $150 \mathrm{~m}$ et le volume de béton s'élève à 940.000 mètres cubes.

\section{The Rosellemel Dam}

J. P. Frey \& H. Legros, engineers.

This dam is located on the higher reaches of the river Doron of Beaufort, in the Alps, close to the Mont Blanc massif. The reservoir has a capacity of $18 \%$ cubic hectometres.

The water is taken along an underground conduit, $13 \mathrm{kms}$ in length, to the power station, which has $500.000 \mathrm{~kW}$ capacity. It utilises a hydrostatic head of $1208 \mathrm{~ms}$, and water flow of $50 \mathrm{~ms}^{3} / \mathrm{sec}$. The total yearly output will ultimately be of the order of $1000 \cdot 16^{6} \mathrm{kWh}$. This energy is obtained from a basin of $272 \mathrm{kms}^{2}$.

This project involved the construction of a $150 \mathrm{~ms}$ high dam. After considering the various alternatives of a gravity, vault, abutment or multiple vault dam, it was finally decided to build a dam consisting of a central, self supporting part, extending on each side with the support of abutments. The design was developed with the aid of reduced scale gypsum
models, which were progressively corrected until the final solution was established.

The upstream side dam has vertical sides. The abutments have a slope of 0.75 , and a maximum thickness of 7.5 ms. The vaulted part of the dam has a maximum slope of 1.05 , and its thickness at the midpoint of the base is $22 \mathrm{~ms}$.

The total length of the dam is $820 \mathrm{~ms}$, its maximum, height is $150 \mathrm{~ms}$, and the volume of concrete employed is $940.000 \mathrm{~ms}^{3}$.

\section{Das Struwelhr Roselend}

J. P. Frey und H. Legros, Ingenieure.

Dieses Stauwehr befindet sich am Oberlauf des Doron de Beaufort in den Alpen, und zwar in der Nähe des Mont BlancMassives. Der Stausee fasst $187 \mathrm{hm}^{3}$.

Die von der Entnahme stammenden Wassermengen werden zum unterirdischen Werke durch einen $13 \mathrm{~km}$ langen Stollen geleitet. Das Werk hat eine Leistung von $500.000 \mathrm{~kW}$; es nützt einen Wasserfall von $1208 \mathrm{~m}$ Höhe und $50 \mathrm{~m}^{3} / \mathrm{s}$ nutzbarer Wetrasen, wofür. Die Erzeugung der elektrischen Energie wird, sobald ${ }^{2}$ rechnen wird.

Wegen der örtlichen Verhältnisse setzte der Abschluss einen Damm von $150 \mathrm{~m}$ Höhe voraus. Nach dem Studium der Lösungen einer Schwergewichtsstaumauer, erleichtert oder mit Widerlagern und vielfachen Gewölben, erwählte man als sich nach beiden Seiten verlängerte in Dämmen, die mit in günstigen Abständen widerständigen Kern haben würde, der den. Die Form ist an einem verkleinerten Gipsmodell studiert worden, das nach jedem Versuch verbessert wurde, bis man das Modell erhielt, welches das beste Verhalten zeigte.

Die Bergseite des Wehrkörpers ist senkrecht. Die Widerlager haben eine Böschung von 0,75 m und eine maximale Dicke von $7,50 \mathrm{~m}$

Was die Wölbung anbetrifft, so beträgt die Böschung 1,05 in der Richtung des Höchstgefälles. Die Gewölbedicke am Grunde und am entsprechenden Scheitelteile beträgt $22 \mathrm{~m}$.

Die Gesamtlänge des Stauwehres beträgt $829 \mathrm{~m}$, seine maximale Höhe $150 \mathrm{~m}$, und das Betonvolumen steigt auf $940.000 \mathrm{~m}^{3}$ an. 